\title{
Identifying dark matter interactions in monojet searches
}

\author{
Prateek Agrawal $^{a}$ and Vikram Rentala ${ }^{b}$ \\ ${ }^{a}$ Fermilab National Accelerator Laboratory, \\ Batavia, IL, 60510, U.S.A. \\ ${ }^{b}$ Department of Physics \& Astronomy, Michigan State University, \\ E. Lansing, $M I$ 48824, U.S.A. \\ E-mail: prateek@fnal.gov, rentala@pa.msu.edu
}

\begin{abstract}
We study the discrimination of quark-initiated jets from gluon-initiated jets in monojet searches for dark matter using the technique of averaged jet energy profiles. We demonstrate our results in the context of effective field theories of dark matter interactions with quarks and gluons, but our methods apply more generally to a wide class of models. Different effective theories of dark matter and the standard model backgrounds each have a characteristic quark/gluon fraction for the leading jet. When used in conjunction with the traditional cut-and-count monojet search, the jet energy profile can be used to set stronger bounds on contact interactions of dark matter. In the event of a discovery of a monojet excess at the $14 \mathrm{TeV}$ LHC, contact interactions between dark matter with quarks or with gluons can be differentiated at the $95 \%$ confidence level. For a given rate at the LHC, signal predictions at direct detection experiments for different dark matter interactions can span five orders of magnitude. The ability to identify these interactions allows us to make a tighter connection between LHC searches and direct detection experiments.
\end{abstract}

KEYwords: Dark Matter and Double Beta Decay, Jet substructure, Jets

ARXIV EPRINT: 1312.5325 


\section{Contents}

1 Introduction 1

2 Dark matter searches with monojets 3

2.1 The effective field theory approach 3

2.2 Searches and limits at the LHC 4

3 Quark/gluon composition of the monojet 5

4 Jet energy profiles $\quad 7$

4.1 Determining the JEPs of a sample of pure quarks/gluons 7

4.1.1 Experimental JEPs 8

$\begin{array}{lll}\text { 4.1.2 Perturbative theory calculation } & 8\end{array}$

4.1.3 Monte-Carlo simulations $\quad 9$

4.2 From individual quark/gluon JEPs to weighted samples 9

5 Methodology 10

5.1 Background 11

$\begin{array}{ll}5.2 \text { Systematic uncertainties } & 11\end{array}$

$\begin{array}{lll}\text { 5.3 Projecting from JEPs to the signal/background fraction } & 12\end{array}$

6 Results 14

$\begin{array}{lll}\text { 6.1 Exclusions at } 8 \mathrm{TeV} \text { LHC } & 14\end{array}$

$\begin{array}{lll}6.2 & \text { Discovery reach and separation of models at } 14 \mathrm{TeV} & 15\end{array}$

$\begin{array}{lll}7 & \text { Implications for direct detection } & 16\end{array}$

$\begin{array}{lll}8 & \text { Discussion and conclusions } & 18\end{array}$

\section{Introduction}

There is overwhelming evidence for the existence of dark matter (DM) from astrophysical and cosmological observations. While its gravitational interactions are well understood, its particle properties such as its mass and other interactions have so far eluded detection.

There are a variety of experiments which are searching for DM particles (see [1] for a review). Direct detection experiments are looking for nuclear recoils from scattering with the DM in our immediate vicinity [2-13]. Indirect detection involves searching for annihilation products of DM from regions of high DM density [14-23]. In this paper we will focus on collider searches for DM [24-38]. At the Large Hadron Collider (LHC), DM can be pair produced at a relatively high rate through interactions with colored particles in the standard model (SM). Associated production of initial state radiation (ISR) jets can then lead to a signature with energetic jets with significant missing transverse energy $\left(E_{T}\right)$, which has been called the "monojet" signal. 
In the limit of a heavy mediator for interactions between the DM particles and the SM, we can use an effective field theory (EFT) description with contact interactions involving the DM and SM quarks and gluons to study the expected monojet signal [39-43]. At the LHC, the interaction is probed at high energies, and the validity of the EFT can be circumspect. One way to derive a more robust bound is to include the mediator in a simplified model framework [44-48]. In addition to the monojet signature, there may also be related signatures of other visible particles produced in association with the DM [49], such as mono-photons [50], mono-W/Z [51-53] and mono-Higgs [54, 55].

There are three main goals for monojet searches that can be phrased in the EFT framework:

1. In the absence of discovery, to set a limit on the suppression scale of new physics operators that couple DM to SM particles.

2. Upon a discovery of an excess at the LHC, to try and identify the new physics operator or combinations of operators that are responsible for the signal.

3. To verify whether the particles escaping the detector and reconstructed as missing transverse momentum in monojet searches constitute the DM of the universe.

In this paper, we will consider two cases: one where DM has contact interactions with quarks and the other where it has contact interactions with gluons. Our main idea is to exploit the fact that these two scenarios for DM interaction and the SM background, each have a characteristic quark/gluon fraction associated with the parton that initiates the monojet. Therefore, any technique which distinguishes between quark- and gluon-initiated jets will effectively discriminate between these DM production operators and also the SM background. In this paper we will use averaged jet energy profiles for such a discrimination. This method can be used to both improve the sensitivity of monojet searches or to identify the type of contact interaction in the event of a discovery of a monojet signal.

The EFT approach simplifies the connection between predictions at the LHC and direct detection experiments. In direct detection experiments, since the DM-nucleon scattering is probed at low momentum transfer, the EFT can be used to reliably calculate the expected signal. However, given a certain monojet cross section at the LHC, different interactions lead to direct detection cross sections that span five orders of magnitude. Therefore, the ability to discriminate between DM interactions with quarks versus interactions with gluons would have major ramifications for direct detection experiments.

For low DM masses $(\lesssim 10 \mathrm{GeV})$, the current experimental limits from the ATLAS and CMS collaborations constrain the suppression scale of the contact interactions much more strongly than current spin-independent direct detection bounds [56, 57]. In the event of a monojet signal in this region, this technique could be used to identify the target search region for future experiments. For higher DM masses, strong limits have been set in direct detection experiments. Thus, given a monojet signal in this region, this technique could help us to evaluate the hypothesis that the particles produced in the monojet signal are the same as the galactic DM. 
This paper is organized as follows. In section 2, we review the current monojet searches at LHC in terms of EFT operators and the current bounds on their suppression scales. In section 3, we will point out how using the quark/gluon fraction of the monojet can improve the bounds or help discriminate between DM contact interactions with quarks versus gluons. Then, in section 4, we will review the concept of jet energy profiles (JEPs) to distinguish between quark and gluon jets. In section 5 , we will describe our methodology for applying the JEPs to monojet searches. In section 6 we will present our results for improving the limits on the suppression scale of the EFT operators using JEPs at the LHC. We will also show how well the two types of DM operators can be distinguished at the $14 \mathrm{TeV}$ LHC assuming discovery of an excess in monojets. In section 7 , we will discuss the implications for direct detection searches for dark matter. Finally, we will conclude in section 8 .

\section{Dark matter searches with monojets}

In this section we discuss the EFT approach to study DM contact interactions with quarks and gluons. We will summarize the current limits placed on the scale of the contact interactions by monojet searches at the LHC.

\subsection{The effective field theory approach}

The EFT approach to DM monojets at the LHC was first studied in [39-43]. We will adopt the same approach as in these papers. We will consider Dirac fermion DM with contact operators of dimensions 6 and 7 that couple pairs of dark matter particles to SM light quarks and gluons respectively.

For simplicity, we will focus on just two operators,

$$
\begin{aligned}
\mathcal{O}_{q} & =\frac{1}{M_{*}^{2}}\left(\bar{q} \gamma_{\mu} q\right)\left(\bar{\chi} \gamma^{\mu} \chi\right), \\
\mathcal{O}_{g} & =\frac{\alpha_{s}}{4 M_{*}^{3}}\left(G_{\mu \nu}^{a} G^{a \mu \nu}\right)(\bar{\chi} \chi) .
\end{aligned}
$$

Here, the normalization has been chosen in accord with ref. [40], where $\mathcal{O}_{q}$ and $\mathcal{O}_{g}$ are referred to as D5 and D11 in their study. For $\mathcal{O}_{q}$ we will assume universal coupling to the up and down type quarks only, which are the ones most relevant for LHC and direct detection searches for the vector-like quark bilinear. We will henceforth refer to these models as QDM and GDM to indicate the coupling of dark matter to quarks and gluons respectively. Each of these models can be parameterized with only two parameters: the dark matter mass, $m_{\chi}$ and the suppression scale, $M_{*}$.

Some alternate choices for the quark contact operators, which we do not consider, involve different bilinears for the quarks. The scalar quark bilinears $\left(\bar{q}_{L} q_{R}\right),\left(\bar{q}_{R} q_{L}\right)$ are expected to be suppressed by the light quark masses and thus the monojet search would give rise to very weak limits on these models. The vector $\left(\bar{q} \gamma_{\mu} q\right)$ and axial vector $\left(\bar{q} \gamma_{\mu} \gamma_{5} q\right)$ bilinears give practically similar limits at collider searches, but the difference is important when we consider direct detection experiments because the former gives rise to spinindependent interactions with nuclei, which are strongly constrained, whereas the latter give spin-dependent interactions, which are weakly constrained. 


\subsection{Searches and limits at the LHC}

We now discuss how monojet searches at the LHC constrain the parameter space of the EFTs for DM. The operators $\mathcal{O}_{q}$ and $\mathcal{O}_{g}$, combined with ISR, can give rise to monojet signatures defined as a high energy jet accompanied by large $E_{T}$ at the LHC. The most dominant irreducible SM background comes from $Z+j$ with $Z \rightarrow \nu \bar{\nu}$. There is also a sub-dominant irreducible background from $W+j$ with $W \rightarrow \tau \nu$, where the $\tau$ decays hadronically. In addition there are reducible backgrounds from $W+j$ with $W \rightarrow \mu \nu$ or $e \nu$, where the lepton is either missed or misidentified as a jet. We will refer to all these sources of SM background as the $V+j$ background.

In order to discover an excess in monojets a cut-and-count strategy is used by the ATLAS and CMS experiments $[56,57]$. The typical $p_{T}$ spectrum of the monojet associated with DM production is more slowly falling relative to the $p_{T}$ spectrum of the background. By choosing different values of the $p_{T}$ and $E_{T}$ cut, the experimental analysis defines different "signal regions" (SR) in which limits are placed on the suppression scale, $M_{*}$ for a given value of $m_{\chi}$. Choosing too low a value of the $p_{T}$ and $E_{T}$ cuts leads to large systematic errors on the predicted number of background events, whereas too high a value of the $p_{T}$ cut reduces the number of events on which to perform statistical analysis.

We will adopt the so-called SR3 region from the ATLAS search, which they use to set limits on the scale $M_{*}$. In this region, the statistical errors are comparable to the systematic errors.

The following cuts are required for event selection in this region:

- $E_{T}>350 \mathrm{GeV}$,

- leading jet with $p_{T}>350 \mathrm{GeV}$ and $|\eta|<2.0$,

- at most two jets with $p_{T}>30 \mathrm{GeV}$ and $|\eta|<4.5$,

- $\Delta \phi\left(\right.$ jet,$\left.E_{T}\right)>0.5$ (second leading jet),

- lepton vetoes.

The resulting limits in the $m_{\chi}-M_{*}$ plane from non-observation of an excess in monojets at the $8 \mathrm{TeV}$ run of the LHC are shown for the operators $\mathcal{O}_{q}$ and $\mathcal{O}_{g}$ in figure 1 . Strictly speaking, the validity of the effective theory description restricts the values of $E \lesssim M_{*}$, where $E$ is the typical energy scale at the monojet vertex. However, even for regions where the effective theory is invalid, the experimental bounds in the $m_{\chi}-M_{*}$ plane can give a qualitative understanding of the bounds on dark matter physics models.

To gain an understanding of the typical kinematics of the monojet signal, we plot contours of the expected signal event distribution as a function of the jet $p_{T}$ and the invariant mass of the DM particles $\left(m_{\bar{\chi} \chi}\right)$ in figure 2 . At the LHC, only the jet $p_{T}$ of the events is observable and the kinematic variable $m_{\bar{\chi} \chi}$ cannot be reconstructed. However, this plot tells us about the typical kinematics of the signal events. By examining this plot we can see that the typical energy transfer at the monojet vertex, $E \simeq \sqrt{m_{\bar{\chi} \chi}^{2}+p_{T}^{2}}$, is close to the current bounds on the effective theory scale $M_{*}$. Thus, the perturbative description of the EFT dark matter theory, with an expansion in $E / M_{*}$, breaks down for some fraction 


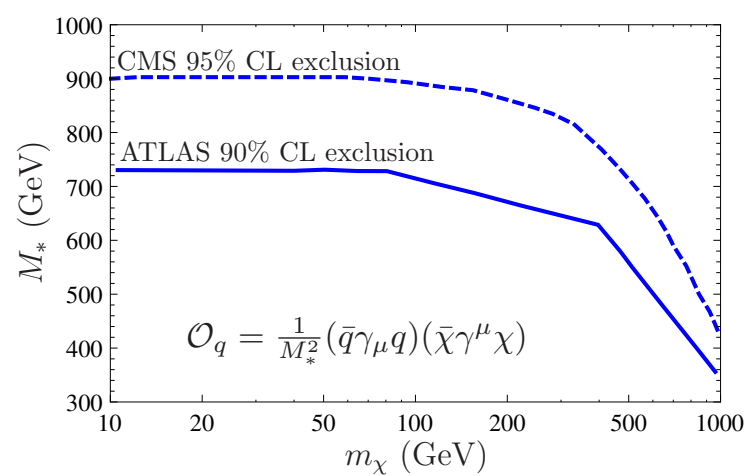

(a) Limit on $M_{*}$ in the QDM model.

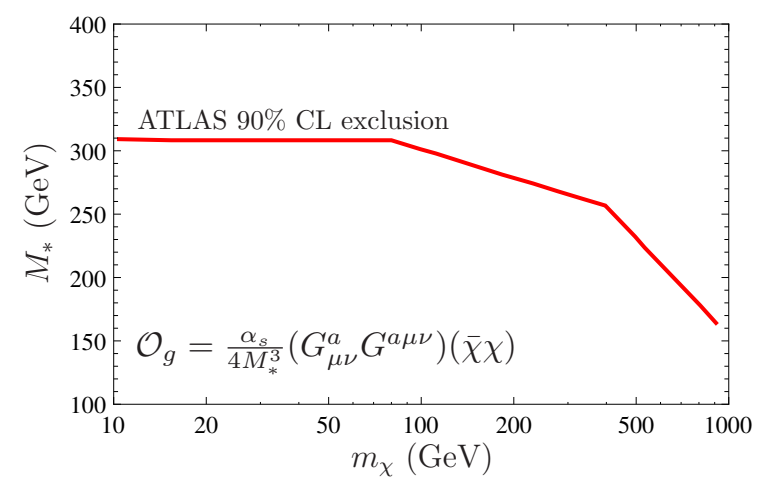

(b) Limit on $M_{*}$ in the GDM model.

Figure 1. ATLAS [56] and CMS [57] exclusions on the suppression scale $M_{*}$ for various dark matter masses from the $8 \mathrm{TeV}$ run of the LHC. ATLAS used $10.5 \mathrm{fb}^{-1}$ of luminosity for their analysis, whereas CMS used $19.5 \mathrm{fb}^{-1}$ of luminosity. CMS has not yet released an official limit for the $O_{g}$ operator.

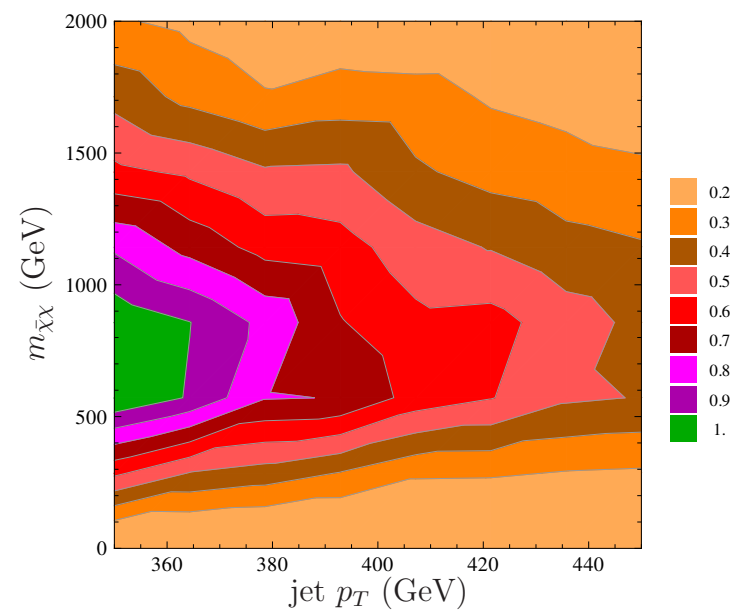

(a) Event kinematic distribution for $\mathcal{O}_{q}$.

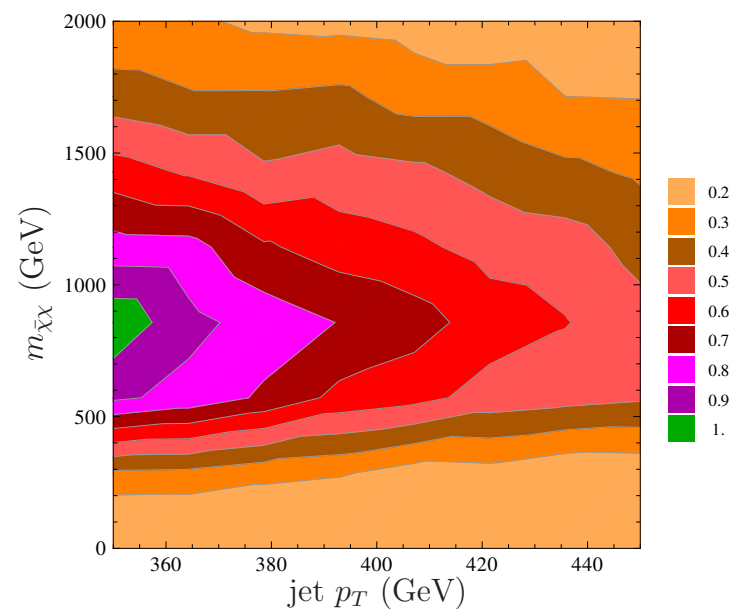

(b) Event kinematic distribution for $\mathcal{O}_{g}$.

Figure 2. Plot of the expected distribution of monojet signal events, at the $8 \mathrm{TeV}$ LHC, as a function of invariant mass of the $\bar{\chi} \chi$ and jet $p_{T}$ in the QDM and GDM models. The overall normalization of the event distribution is arbitrary. We can see that most events are produced with a large center of mass energy $E \simeq \sqrt{m_{\bar{\chi} \chi}^{2}+p_{T}^{2}}$, close to the current bound on the scale of the effective operators, $M_{*}$ in each of the respective theories.

of the events as higher dimensional operators become somewhat important. Nevertheless, we will continue to use the EFT description ignoring the higher order corrections and it will not affect our main conclusions.

\section{Quark/gluon composition of the monojet}

It is interesting to consider the composition of quarks/gluons in monojet signals and backgrounds. This can be parameterized as a gluon fraction $f_{g}$. The typical gluon fractions 


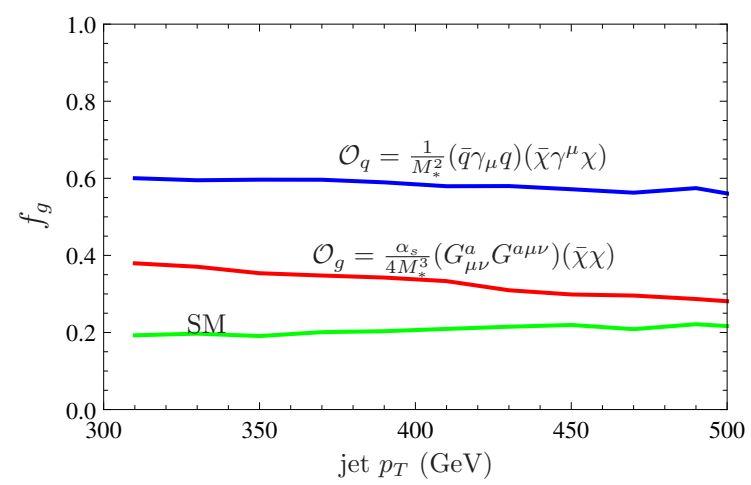

(a) Gluon fraction vs $p_{T}$.

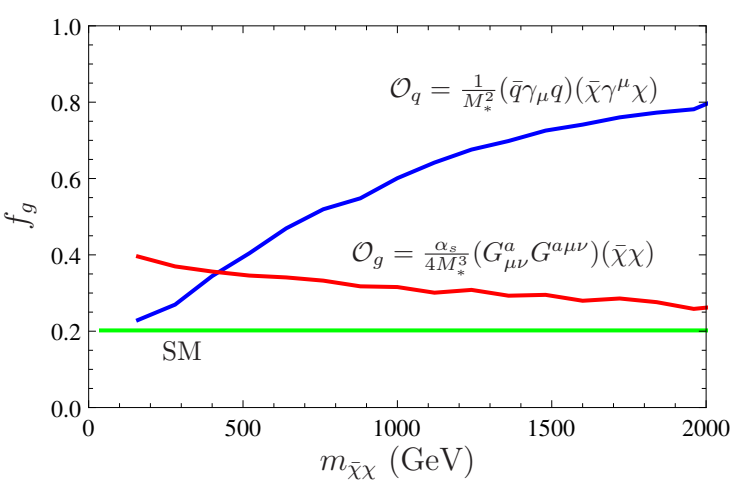

(b) Gluon fraction vs $m_{\bar{\chi} \chi}$.

Figure 3. Plots of the gluon fractions $\left(f_{g}\right)$ at the $8 \mathrm{TeV}$ LHC as (a) a function of jet $p_{T}$ for the monojet signal and the SM background and (b) $m_{\bar{\chi} \chi}$ for the monojet signal. In (b) the SM $\left(f_{g}\right)$ value is shown only for reference. The differing gluon fractions of the signals and the background as a function of the jet $p_{T}$ suggest that experimental separation based on this variable could allow for better separation of signal and background than a counting experiment alone. In the event of a discovery this technique could also allow us to identify the operator that is responsible for the signal.

for a sample of monojets generated from a pure signal sample from $\mathcal{O}_{q}, \mathcal{O}_{g}$ and a pure background sample from $V+j$ are shown as a function of the jet $p_{T}$ in figure 3 a and as a function of $m_{\bar{\chi} \chi}$ in figure $3 \mathrm{~b}$.

We note some interesting features of the gluon fractions,

- the QDM sample at all jet $p_{T} \mathrm{~s}$ is slightly richer in gluons than in quarks $\left(f_{g} \simeq 0.6\right)$, while the $V+j$ sample is quark rich $\left(f_{g} \simeq 0.2\right)$. The $V+j$ and QDM processes are generated by similar processes with similar diagrams, so this result seems surprising at first. We shall explain this discrepancy in the gluon fractions below.

At the parton level there are two main processes that contribute to $V /(\bar{\chi} \chi)+j$ are $q \bar{q} \rightarrow V /(\bar{\chi} \chi) g$ and $q g \rightarrow V /(\bar{\chi} \chi) q$. For fixed energies for the initial partons, in both the SM and QDM monojets, the rate for the $q \bar{q}$ initiated process typically dominates the $q g$ initiated process by a factor of a few.

However, the main distinction is that the QDM kinematics is controlled by $m_{\bar{\chi} \chi}$ (figure 2) and is probed at high momentum fraction $(x)$ for the partons, whereas the $V+j$ kinematics is controlled by the mass of the $\mathrm{W}$ and $\mathrm{Z}$ bosons $\left(m_{W} / m_{Z}\right)$ and is probed at a relatively low value of $x$. This leads to the $q g$ initiated process being highly enhanced for $V+j$ due to the large gluon parton density function (pdf), which leads to the SM monojet being quark rich. However, the QDM monojet is produced at larger $x$ and the enhancement of the gluon pdf relative to the sea $\bar{q}$ pdf is smaller and both processes contribute similarly leading to roughly similar quark and gluon fractions. We can see this effect in figure 3b, where the gluon fraction for the QDM signal starts of the same as the SM when $m_{\bar{\chi} \chi} \simeq m_{W} / m_{Z}$ (low $x$ values) and rises strongly with the mass of the $\bar{\chi} \chi$ (high $x$ values). 
- The GDM sample starts off at $\left(f_{g} \simeq 0.4\right)$ near the $p_{T}$ cut threshold and drops to $\left(f_{g} \simeq 0.2\right)$ for higher $p_{T} \mathrm{~s}$. The GDM production takes place through the processes $g g \rightarrow \bar{\chi} \chi g$ and $g q \rightarrow \bar{\chi} \chi q$. Here the $g q$ initiated process dominates for fixed partonic energies. However, at low values of $p_{T}$ or $m_{\bar{\chi} \chi}$ the gluon pdfs lead to the $g g$ process being enhanced and hence $f_{g} \simeq 0.4$. At large values of $p_{T}$ or $m_{\bar{\chi} \chi}$ the $g q$ process dominates due to the valence quark pdf being probed at high $x$ and we see that the monojet sample becomes quark rich $\left(f_{g} \simeq 0.2\right)$.

The characteristic distinction between $f_{g}$ in each of the three cases strongly suggests that identification of the gluon fraction of the monojet sample is a good way to enhance sensitivity in monojet searches by providing an additional handle to discriminate signal from background. The distinction can also be used to discriminate between different signal hypotheses once an excess in monojets is discovered. We will study the use of jet energy profiles (JEPs) in the next section to see how the gluon fractions can be probed.

\section{Jet energy profiles}

We now describe the technique of using jet energy profiles (JEPs) to identify the gluon fraction of a jet sample. We will consider anti- $k_{T}$ jets [58] with a cone size $R=0.7$ as measured in the rapidity-azimuth $(\eta-\phi)$ plane. The JEP, $\psi(r)$, for an individual jet is defined as the fraction of jet $p_{T}$ inside a subcone of size $r$ relative to the total jet $p_{T}$,

$$
\psi(r)=\frac{\int_{0}^{r} \frac{d p_{T}}{d r^{\prime}} d r^{\prime}}{\int_{0}^{R} \frac{d p_{T}}{d r^{\prime}} d r^{\prime}},
$$

where the denominator is just the total $p_{T}$ of the jet. The JEP is defined so that $\psi(0)=0$ and $\psi(R)=1$. Gluon initiated jets are expected to spread more due to more radiation and thus have a slowly rising JEP. Quark initiated jets on the other hand radiate less, and thus accumulate a larger fraction of their energy for fairly small $r$ and have a quickly rising JEP. A typical quark/gluon profile is shown in figure 4a.

For an individual quark/gluon the JEP fluctuates widely due to a Sudakov tail as shown in figure $4 \mathrm{~b}$. The characteristic shape and spread of the JEP also depends on the jet $p_{T}$. This variance makes an individual quark/gluon jet difficult to tag. However, on average, a sample of pure quark or gluon jets can be distinguished fairly well statistically as we shall see.

\subsection{Determining the JEPs of a sample of pure quarks/gluons}

There are several different approaches that one could take towards determining the average JEP of a sample of pure quark or gluon events.

1. Experimental: data driven approach,

2. Theory: perturbative QCD (pQCD) calculation,

3. Simulation: Monte-Carlo shower and hadronization generators. 


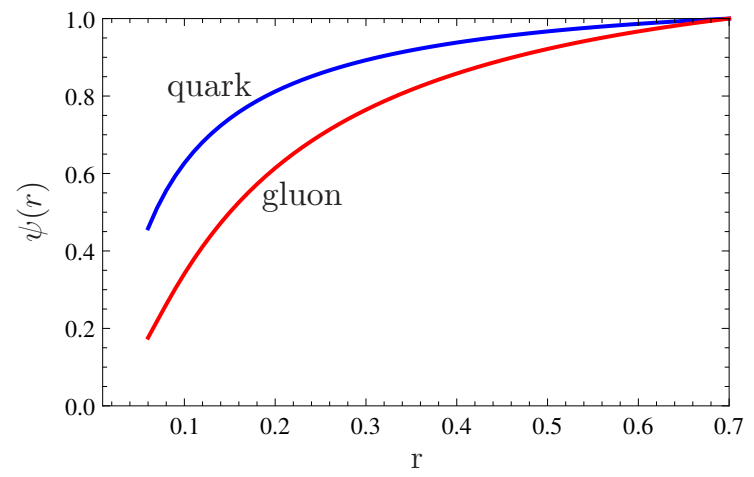

(a)

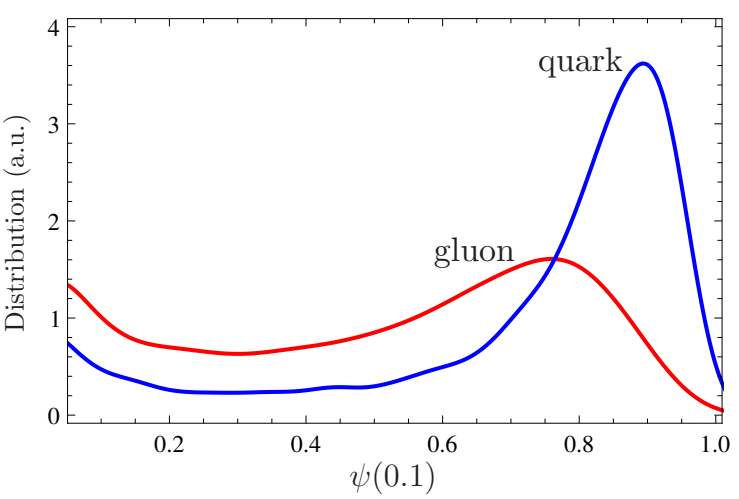

(b)

Figure 4. (a) Typical quark and gluon jet energy profiles, $\psi(r)$. Quarks typically radiate less and have a more steeply rising JEP as compared to gluons. (b) Distribution (with arbitrary normalization) of $\psi(0.1)$ for quark and gluon jets generated in PYTHIA 8 [59]. Individual jets may fluctuate in their profiles significantly. We can see here the Sudakov tail of the radiation pattern.

The best approach to determining the JEPs is to use all three techniques in conjunction. We will discuss the merits and demerits of each approach below.

\subsubsection{Experimental JEPs}

JEPs have been measured at the Tevatron CDF experiment [60] and at the LHC by both ATLAS [61] and CMS [62]. Assuming factorizability (i.e. universal behavior) of quark/gluon jets of a given $p_{T}$, we could measure the average quark/gluon profile in control samples of pure (or theoretically well known fractions of) quark/gluon jets. The experimental JEPs have the advantage of having very small error bars and thus allow for a very precise determination of the average quark/gluon jet profile for a given $p_{T}$. However, factorizability must be proven theoretically to guarantee the validity of using calibrated quark/gluon JEPs in a control sample to match the behavior of quark/gluon JEPs initiated from a different hard process. Theoretical calculations may also be needed to extrapolate the behavior of JEPs with $p_{T} \mathrm{~S}$ outside of the experimental control regions.

\subsubsection{Perturbative theory calculation}

The JEPs can be calculated using the techniques of pQCD. At next-to-leading order (NLO) the profiles were found to overshoot the data [63] at small $r$, where the logarithmic corrections $\alpha_{s} \log (R / r)$ are important and need to be resummed. The fixed order results are thus inappropriate for comparison with experiment. The next-to-leading-logarithm (NLL) terms of the form $\alpha_{s}^{n}(\log (R / r))^{2 n}$ and $\alpha_{s}^{n}(\log (R / r))^{2 n-1}$ need to be resummed to all orders in $\alpha_{s}$ to obtain a finite answer. The resummed JEPs were calculated in [63,64]. Very good agreement was found between the experimental data and the resummation calculation for a wide range of jet $p_{T} \mathrm{~s}$. It was also theoretically confirmed that a gluon jet is broader than a quark jet with the same $p_{T}$. The NLL resummed JEPs capture the behavior of the experimental JEPs extremely well and demonstrate factorizability. 
In order to match the theoretical prediction to the experimental results, it is necessary to fix some (arbitrary) scale parameters which are introduced into the pQCD calculations to estimate the effects of the sub-leading logarithmic contributions. Varying the scale parameters allows us to estimate the error on the theoretical calculation. The theoretical error on the JEP is large compared to the experimental uncertainties on JEPs. However, upon calibrating to the data in a known control region, the scale parameters can be fixed and the resulting JEPs can be extrapolated outside of the control regions of experiments.

The resummed theory calculation only predicts the average JEP for a sample of quarks and gluons. By itself, it does not show the fluctuations that would be needed to determine the statistical uncertainty of the JEP at an experiment.

\subsubsection{Monte-Carlo simulations}

Monte-Carlo shower and hadronization generators such as PYTHIA [59] can also be used to produce the JEPs. The predictions of the Monte-Carlo are notoriously tune-dependent and need to be calibrated to match the data, for e.g. PYTHIA tune $\mathrm{A}^{1}$ can describe the observed JEPs well. The advantage of the Monte-Carlo method is that it allows us to generate event samples that can be used to predict the statistical uncertainty of the JEP.

As theorists we have access only to the latter two methods. In order to calculate the central value of the average JEPs, we will use the results of the NLL resummation calculation. We fix the values of the integration constants by matching the theory calculation to Tevatron data. In order to estimate the statistical uncertainty, we will use the default tune of PYTHIA 8 to generate fluctuations and transpose the error bars obtained in this manner onto the pQCD JEPs.

\subsection{From individual quark/gluon JEPs to weighted samples}

As noted above, there are large Sudakov tails in the JEP distribution for individual quarks/gluons. In order to reduce sensitivity to the shape of this distribution, we will work with the profile averaged over a sample of jets. The immediate advantage is that the average profile is Gaussian distributed if the sample size is sufficiently large, due to the central limit theorem. The error bars on the profiles then scale simply with the size of the sample $(N)$ as $1 / \sqrt{N}$.

It is also convenient to work at the level of individual models, instead of a pure quark or gluon sample. The pQCD calculation provides the expected average JEP for pure samples of quarks or gluons in a given $p_{T}$ bin. Thus, given the quark/gluon composition in respective $p_{T}$ bins from the model, we can predict the average profile for a sample. The average profile is a weighted average of quark and gluon JEPs, with the weight depending on the underlying process, parton distribution functions, collider energy and jet $p_{T}$. For the processes we consider, the profile has a very mild dependence on the $p_{T}$ of the jet, due to both $p_{T}$-dependent QCD effects as well as different quark-gluon fractions in different $p_{T}$ bins (see figure 3a).

\footnotetext{
${ }^{1}$ PYTHIA-Tune A. Monte Carlo samples are generated using the following tuned parameters in PYTHIA: $\operatorname{PARP}(67)=4.0, \operatorname{MSTP}(82)=4, \operatorname{PARP}(82)=2.0, \operatorname{PARP}(84)=0.4, \operatorname{PARP}(85)=0.9, \operatorname{PARP}(86)=0.95$, $\operatorname{PARP}(89)=1800.0, \operatorname{PARP}(90)=0.25$.
} 
Averaging over $p_{T}$ bins, we arrive at a single profile which we call the "expected average profile" (EAP),

$$
\psi(r)_{\mathrm{EAP}}=\int\left(\frac{d N_{q}}{d p_{T}} \psi_{q}\left(r, p_{T}\right)+\frac{d N_{g}}{d p_{T}} \psi_{g}\left(r, p_{T}\right)\right) d p_{T} /\left(N_{q}+N_{g}\right) .
$$

Here, $N_{q}$ and $N_{g}$ are the number of quarks and gluons initiating the jets in the sample. $\psi_{q}\left(r, p_{T}\right)$ and $\psi_{g}\left(r, p_{T}\right)$ are the average quark and gluon profiles for jets of a particular $p_{T}$, which can be calculated from the pQCD resummation technique. The statistical fluctuations in the average JEP are controlled by both the statistical fluctuations of the gluon fraction of the sample and the statistical fluctuations of the profiles of individual quark and gluons jets (figure 4b). The size of these statistical fluctuations can be determined by Monte-Carlo methods which simulate the hard process (which calculates the fluctuation in the quark/gluon fractions) and the soft/collinear processes (which leads to fluctuations in the individual quark/gluon JEPs).

\section{Methodology}

We now discuss the application of JEPs to monojet searches. As discussed, we will consider two EFTs for dark matter, labeled QDM and GDM, corresponding to the following interactions respectively

$$
\begin{aligned}
\mathcal{O}_{q} & =\frac{1}{M_{*}^{2}}\left(\bar{q} \gamma_{\mu} q\right)\left(\bar{\chi} \gamma^{\mu} \chi\right), \\
\mathcal{O}_{g} & =\frac{\alpha_{s}}{4 M_{*}^{3}}\left(G_{\mu \nu}^{a} G^{a \mu \nu}\right)(\bar{\chi} \chi) .
\end{aligned}
$$

In section 3, we saw that the QDM and GDM models and the SM background each have characteristic quark/gluon fractions. In this section we will separate the different DM models and the SM background using JEPs. We will benchmark against the $10.5 \mathrm{fb}^{-1}$ ATLAS results [56], since their analysis sets limits for both the QDM and GDM EFTs that we are interested in.

The QDM and GDM models are each characterized by two parameters, the dark matter mass, $m_{\chi}$ and the suppression scale of the effective operator, $M_{*}$. The dark matter mass affects the production cross section, as well as the $p_{T}$ spectrum of the radiated jets in monojet searches. We simulate samples for dark matter masses in the range 10 to $1000 \mathrm{GeV}$. Within the region of validity of the EFT, the suppression scale $M_{*}$ only affects the total rate of production. Therefore, predictions for varying values of $M_{*}$ can be easily obtained by scaling the expected number of events.

For each signal + background model, we have two observables to calculate: the expected number of events $(\mathrm{N})$ and the EAP of the monojet $\psi(r)_{\text {EAP. These observables can }}$ then be compared to the corresponding expectations from the background only hypothesis.

We will evaluate the advantage of including the JEP over just a cut-and-count experiment in two contexts:

1. Improvement of the exclusion limits on $M_{*}$ as a function of $m_{\chi}$ for $8 \mathrm{TeV}$ monojet searches.

2. Higher discovery reach, and the power to distinguish the QDM and GDM EFTs at the $14 \mathrm{TeV}$ LHC. 
In order to determine our ability to separate $N$ and $\psi(r)_{\text {EAP }}$ under different hypotheses, we need to estimate the errors on these quantities. Below, we will discuss how we obtain the statistical errors on these quantities by conducting pseudo-experiments.

We simulate monojet samples for the QDM + SM, GDM + SM and SM only scenarios at the parton level with MadGraph5 [65] for a range of dark matter masses. We then shower the events using PYTHIA and use SpartyJet [66] (a wrapper for FastJet [67]) to perform the jet clustering using the anti- $k_{T}$ algorithm. The cuts on the event sample for the SR3 region (see section 2) are then applied to these clustered events. For a given luminosity we can now predict the expected number of events $N_{\exp }$ and its statistical fluctuations $\left(\sqrt{N_{\exp }}\right)$ in each model.

We then consider the leading $p_{T}$ jet for each event in the sample. We construct the jet energy profile $\psi(r)$ of the leading jet by sampling at seven different values of $r=0.1 \ldots 0.7$, as would be expected in a realistic calorimeter simulation. From this collection of JEPs we calculate the average JEP. By running many pseudo-experiments we can also predict the statistical fluctuation on $\psi(r)$ for each value of $r$.

The average JEP for a large number of events should in principle reproduce the EAP. As mentioned in the previous section, this determination is subject to large uncertainties from the PYTHIA tune. We will therefore use the theory prediction to derive the EAP in each model by constructing it from the pQCD calculation and the leading order prediction of the quark/gluon fractions at different $p_{T} \mathrm{~s}$ from MadGraph5. We will, however, retain the statistical error bars from PYTHIA on the JEPs.

Before moving on to our results, we present a few important details for our analysis in this section.

\subsection{Background}

The dominant SM background arises from $Z / W+j$, with the $Z$ decaying to neutrinos, and the $W$ decaying to hadronic taus, or to leptons which are either missed by the detector or misidentified as jets. This latter component of the background is sensitive to detector effects which are hard to model. However, the gluon fraction of the associated jet is nearly identical for both the $Z+j$ and the $W+j$ background. Therefore, we choose to model the entire background as a scaled-up $Z+j$ background. For the $8 \mathrm{TeV}$ analysis, we simulate the background by generating the $Z+j$ background with all the relevant cuts outlined in section 2 , and then scale the simulated cross section to agree with the expected number of events for the $10.5 \mathrm{fb}^{-1}$ ATLAS study [56]. This scaling also includes the k-factor for the full $V+j$ background. For $14 \mathrm{TeV}$ projections, we simulate $Z+j$ at the $14 \mathrm{TeV} \mathrm{LHC}$, and apply the same scaling factor as in the $8 \mathrm{TeV}$ case, to get the total background prediction.

The really crucial ingredient for background modeling comes from the systematic uncertainties. We discuss this in detail in the next subsection.

\subsection{Systematic uncertainties}

We will now discuss the systematic uncertainties on each observable in our analysis - the total number of events, and the jet energy profile. 
The systematic error on the total number of events is controlled by systematics on the SM background rate as well as on the QDM/GDM signal rate. For the $8 \mathrm{TeV}$ ATLAS analysis [56], the background was estimated from a combination of data driven and Monte Carlo simulations. Therefore, the systematic uncertainty on the background has contribution from the control region statistical uncertainty, the simulation statistical uncertainty, and uncertainty in the jet energy scale and other uncertainties, leading to a $7 \%$ uncertainty on the background rate in the relevant signal region. For our $8 \mathrm{TeV}$ exclusion analysis, we will add this systematic error in quadrature with our statistical errors when modeling the fluctuations of the background. The corresponding systematic uncertainties at $14 \mathrm{TeV}$ needs a detailed study. We will choose $5 \%$ systematic on the background rate as a benchmark at $14 \mathrm{TeV}$. For a detailed theoretical study of background systematics, see [68].

We do not include systematic uncertainties for the signal. While the number of signal events itself is subject to systematic uncertainties, these are traditionally accounted for by presenting exclusions for $\pm 1 \sigma$ systematic variation of the predicted cross section. In our study we only present the limits for the nominal signal prediction.

The systematic uncertainty on our modeling of the EAP is important to consider. By examining our formula for the EAP, eq. (4.2), we see that there are several sources of systematic uncertainty. Firstly, we need to know the gluon fraction as a function of the jet $p_{T}$. We are only using the leading order prediction for $f_{g}\left(p_{T}\right)$, but NLO predictions for both the signal and background are needed to make a precise prediction on this fraction. We will neglect this systematic because typically the ratios of rates have a smaller uncertainty than the total rate itself. This is a relevant issue and warrants further study in future work. Secondly, the average JEP for a pure quark or gluon sample is determined from the pQCD NLL calculation calibrated to data to fix the integration constants. In this kind of data driven determination, the statistical uncertainty of the control sample will contribute, but this error as mentioned earlier is extremely small and can be ignored. However, outside of the control $p_{T}$ regions, the errors from the theory calculation can contribute to the systematic uncertainty on the JEP. Finally, from experimental considerations, the measured JEP at the $14 \mathrm{TeV}$ LHC will be subject to increased pile-up activity which implies that there would be much more extraneous radiation which could potentially get clustered with jets arising from the hard process, which will tend to smear the distinction between quark and gluon jets (see [69] for one strategy for dealing with pile-up). This source of systematic needs to be studied in detail once the ATLAS and CMS experiments start collecting data at $14 \mathrm{TeV}$.

In summary, we will neglect all systematic contributions to the uncertainty on the EAPs for the pure GDM, pure QDM and pure SM samples, and we will only estimate the statistical errors on these EAPs.

\subsection{Projecting from JEPs to the signal/background fraction}

For each hypothesis (QDM + SM, GDM + SM and SM only), we can determine the average JEP for the monojet sample and its statistical fluctuations. We would like to project this average JEP onto a single variable that can be compared for these different models. 
We define a one-variable parametrization $f$ of the profile, which interpolates between a SM EAP $(f=0)$ and a QDM EAP $(f=1)$. For a given sample, $f$ is defined as the best-fit parameter of the average profile to the following expression,

$$
\psi_{f}(r)=f \psi_{\mathrm{EAP}}^{\mathrm{QDM}}(r)+(1-f) \psi_{\mathrm{EAP}}^{\mathrm{SM}}(r) .
$$

For a sample of QDM + SM events $f$ can be approximately understood as the fraction of signal events in the sample averaged over all $p_{T}$ bands. It is worth noting that even for the GDM model, we evaluate $f$ as interpolating between the QDM and the SM profile. This is due to the fact that the gluon fraction for the GDM sample resembles more closely the gluon fraction of the SM sample, and hence the profiles are not well separated from each other.

The fluctuations in the pseudo-experiment average JEPs can then be translated into fluctuations in $f$. The expected value of $f$ will also have systematic uncertainties due to the systematic errors on the JEP (which we neglect) and systematic uncertainty in the prediction of the number of background events (which we include).

We have now parameterized every pseudo-experiment monojet sample by two variables, its JEP projected on to the parameter $f$, and the total number of events $N$. Since we have underlying distributions for each component, we can now make expected distributions in the $f$ - $N$ plane for each model. We do so for all three different hypotheses we wish to compare - QDM + SM, GDM + SM and pure SM background.

The central values of $N$ and $f$ in the signal cases depend both on $m_{\chi}$ and $M_{*}$. We generate a number of pseudo-experiments using the above mentioned distributions. For each pseudo-experiment we retain the value of $N$ (the total number of events), and also obtain the best fit value for $f$. We hence get a histogram in the $f$ - $N$ plane for each of the three hypotheses.

The overlap of these two histograms is indicative of sensitivity of the experiment to the specific value of $M_{*}$. Quantitatively, the expected exclusion or discovery confidence can be calculated using for instance the Pearson's $\chi^{2}$-test,

$$
\chi^{2}=\frac{\left(\mu^{N_{\exp }}-\mu^{N_{\text {obs }}}\right)^{2}}{\left(\sigma^{N_{\exp }}\right)^{2}}+\frac{\left(\mu^{f_{\exp }}-\mu^{f_{\text {obs }}}\right)^{2}}{\left(\sigma^{f_{\exp }}\right)^{2}}
$$

where $\mu, \sigma$ denote the mean and standard deviation obtained for observed and expected (from a given hypothesis) quantities. For the emulation of the counting experiment, we use the $\chi^{2}$ value derived from the number of events alone. Similarly, for the separation of hypotheses, we use the $\chi^{2}$ value derived from only $f$. Technically, the parameters $f$ and $N$ are correlated, so treating them as uncorrelated over-estimates the error. However, we will see that the correlation in the relevant region of parameter space is small.

For exclusion at $8 \mathrm{TeV}$, we use the number of events observed in the ATLAS analysis [56] for $\mu^{N_{\text {obs }}}$, and assume $\mu^{f_{\text {obs }}}$ to be at the SM prediction. We can then calculate the $\chi^{2}$ statistic for excluding each model, QDM + SM, or GDM + SM.

For discovery of each model at $14 \mathrm{TeV}$, we assume for $\mu^{N_{\text {obs }}}$ and $\mu^{f_{\text {obs }}}$ values predicted by the corresponding model. In this case, we calculate the $\chi^{2}$ statistic for excluding the SM hypothesis. The value of $M_{*}$ which allows us to rule out the SM at $5 \sigma$ level is the discovery reach of the analysis. 
In the next section we will use the parameters $f$ and $N$ in conjunction using the $\chi$-squared test statistic to enhance the sensitivity of monojet searches for both exclusion limit setting and discovery. In the event of a discovery of an excess in monojet searches, the operator is clearly not determined uniquely. We will also present our results for separating the QDM hypothesis from the GDM hypothesis in the event of such a discovery using the parameter $f$.

\section{Results}

We will now present our results for the expected enhanced sensitivity of monojet searches when using JEPs. Our results will address two different aspects of the monojet search. We study the improvement in the exclusion limits placed by monojet searches when using JEPs at the $8 \mathrm{TeV}$ LHC. We use the ATLAS $10.5 \mathrm{fb}^{-1}$ search [56] as a benchmark for this case. We also consider the discovery reach at the $14 \mathrm{TeV}$ LHC and study the extent to which the two different models (QDM and GDM) of dark matter can be separated in the event of a discovery.

\subsection{Exclusions at $8 \mathrm{TeV}$ LHC}

As shown in figure 1, current ATLAS and CMS analyses put constraints on the suppression scale of various dark matter effective operators, parameterized as $M_{*}$. In this section we will show the expected improvement in the ATLAS exclusion limit when using JEPs.

In figure 5a, we plot the $95 \%$ exclusion limits for the QDM and GDM models. We show the limits obtained by just the cut-and-count analysis of the experiment, which agrees well with the limits set by the experimental analysis. We also present $95 \%$ exclusion limits obtained from a combined $\chi^{2}$ test using the JEPs parameterized by $f$ and the number of events $N$. We use observed and expected background events published in [56]. We assume that the observed jet energy profile is consistent with the SM prediction when setting the exclusion.

We see that for the QDM model the limits obtained are somewhat more stringent. This is due to a strong separation in the JEP for the background only sample and the SM + QDM sample as shown in figure 5b. The average JEP in the case of GDM is much closer to the background, and hence there is no significant improvement in the limits in this case.

The improvement in limits is moderate. This is partially due to the fact that the current limits are set in the regime where the statistical and the systematic uncertainty on the expected number of background events is comparable, and $S / B \ll S / \sqrt{B}$. Therefore, the overall JEP of signal + background is dominated by the background (i.e. $V+j$ ) profile $(f \simeq 0)$. Further, the dark matter event rate is highly sensitive to the value of $M_{*}$, and therefore including the JEP leads to only a marginal increase in the sensitivity to this parameter.

Nonetheless, measuring the value of $f$ constitutes an independent check of the SM background hypothesis. A value of $f$ which is consistent with zero points to an absence of an appreciable dark matter signal, even without a precise prediction of the background. Therefore, in searches where the number of background events is subject to large systematic uncertainty, the JEP measurement can provide valuable complementary information. 


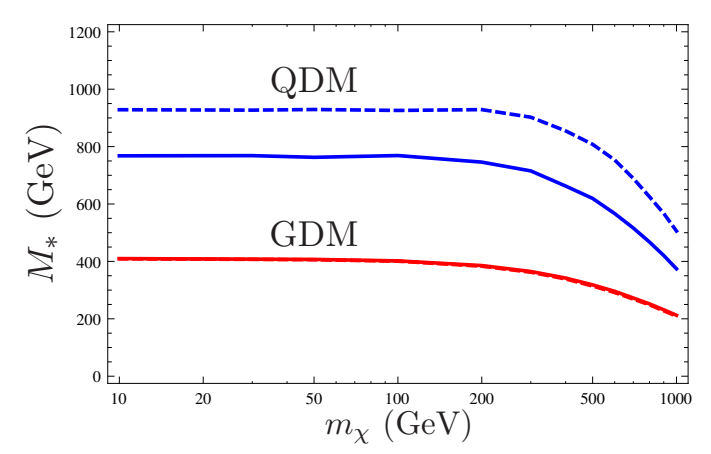

(a)

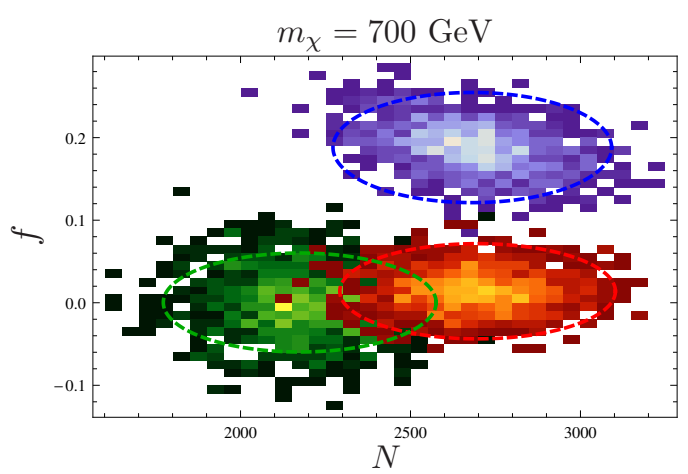

(b)

Figure 5. Exclusion limits from our monojet analysis at the $8 \mathrm{TeV}$ LHC at $10.5 \mathrm{fb}^{-1}$. Left: $95 \%$ limits on $M_{*}$ based on the counting experiment (solid lines) and the joint exclusion limits using both the counts and the JEPs (dashed lines). Limits are shown for both dark matter models, QDM (blue) and GDM (red). Right: a normalized histogram with $1 \sigma$ contours of pseudo-experiment distributions in the $f, N$ plane for dark matter mass $m_{\chi}=700 \mathrm{GeV}$. The hypothesis considered are pure SM background (green), QDM + SM (blue) and GDM + SM (red). The values of $M_{*}$ for each signal model were chosen to be at the bound derived from the counting experiment (solid lines in the left figure).

\subsection{Discovery reach and separation of models at $14 \mathrm{TeV}$}

We present the projected sensitivity of monojet searches at the $14 \mathrm{TeV}$ LHC at two benchmark luminosities, $10 \mathrm{fb}^{-1}$ and $100 \mathrm{fb}^{-1}$. We use the same selection cuts as in the $8 \mathrm{TeV}$ analysis. At $14 \mathrm{TeV}$, the production cross sections are larger by about an order of magnitude relative to $8 \mathrm{TeV}$. Consequently, the relative statistical uncertainty is much smaller. We present our results with a benchmark $5 \%$ systematic error on the number of background events. Note that with the same $p_{T}$ cuts as in the $8 \mathrm{TeV}$ analysis, the $5 \%$ systematic error on the number of events at $14 \mathrm{TeV}$ completely dominates the statistical error already at $10 \mathrm{fb}^{-1}$ of luminosity.

We show the limits obtained by a counting experiment at the $14 \mathrm{TeV}$ LHC in figure 6 . Since the systematic error dominates, increasing the luminosity from $10 \mathrm{fb}^{-1}$ to a $100 \mathrm{fb}^{-1}$ does not increase the discovery reach in a counting experiment. However, in this case the discovery reach can be greatly enhanced by including the JEP, especially at higher luminosity. This is due to the fact that the signal + background models show a remarkable separation in $f$ from the background-only model even when the separation in $N$ is poor due to systematic error dominance. Further, the QDM + SM and the GDM + SM hypotheses are also very well separated. This separation can be explicitly seen in figure 6 .

Thus, our main point is that a conventional counting experiment is systematic dominated and would benefit immensely from the inclusion of the jet energy profiles. In practice, we would expect to compensate for the large systematic error by a redefinition of the signal region by using a larger $p_{T}$ cut, so as to make the statistical and the systematic errors comparable. In this scenario, the improvement in discovery reach offered by the JEPs might be slightly less drastic. For contrast, we also present results for a scenario with zero systematic errors in figure 7. This is obviously an unrealistic assumption, and we merely 

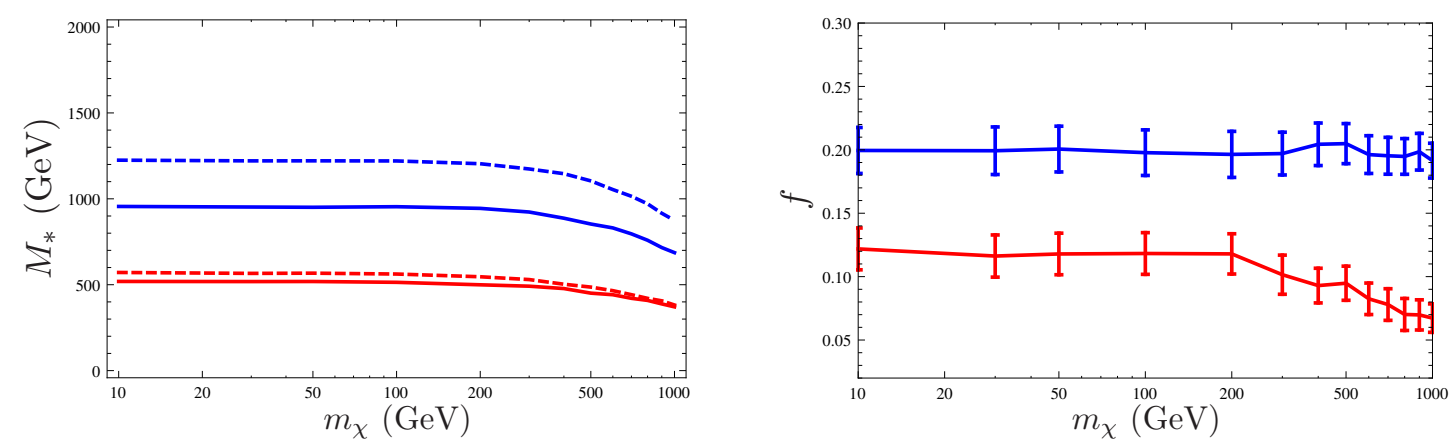

(a) $10 \mathrm{fb}^{-1}$
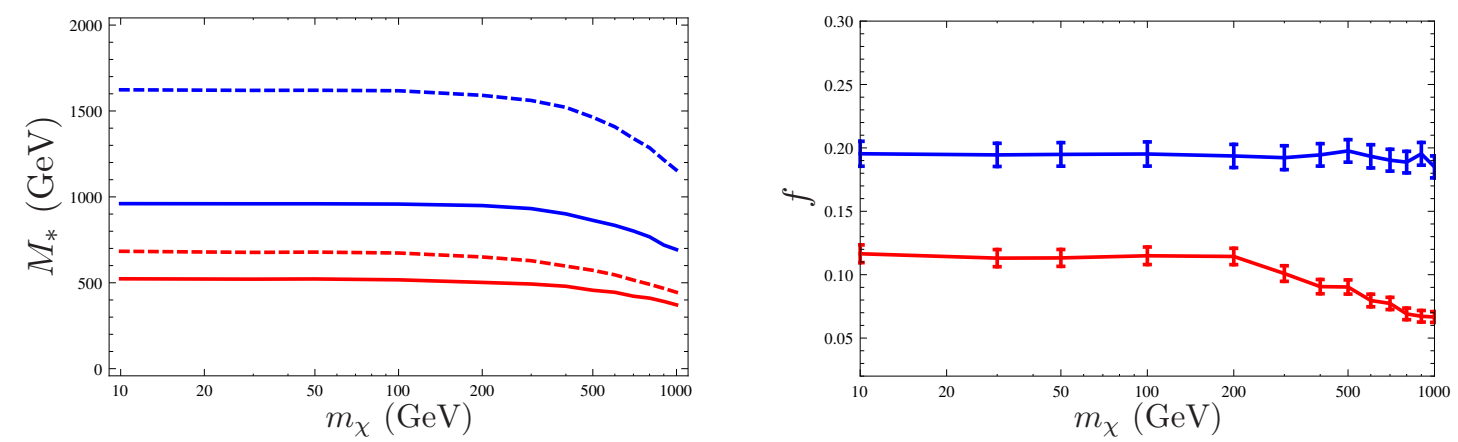

(b) $100 \mathrm{fb}^{-1}$

Figure 6. $5 \sigma$ discovery potential and model separation at $14 \mathrm{TeV}$ at a) $10 \mathrm{fb}^{-1}$ and b) $100 \mathrm{fb}^{-1}$ with a $5 \%$ systematic uncertainty on the background prediction. Left: we show the discovery reach for the parameter $M_{*}$ based on the counting experiment (solid lines) and the joint discovery by both the counting and the jet energy profiles (dashed lines). Limits are shown for both operators, QDM (blue) and GDM (red). Right: we also show the level of separation achieved between the two operators in the presence of background, QDM (blue) and GDM (red), when $M_{*}$ is fixed at the $5 \sigma$ discovery limit for each mass.

include it to show the degradation of the improvement in discovery reach using JEPs. We see that in this case the discovery reach is not improved by adding JEP. However, even in this case we see that we can still comfortably differentiate between the two different dark matter operators. Therefore, the JEPs are effective and crucial in differentiating different DM operators in monojet searches.

We reiterate here that we have not included a systematic error on the ideal prediction of the JEP (EAP) for a given model. We expect that our main conclusion of an improvement in the discovery reach and separability of the DM operators will still hold true even after these systematics are included. The detailed study of systematic uncertainties on the JEPs is left to future work.

\section{Implications for direct detection}

In this section we describe how a discovery in monojet searches would relate to predictions for direct detection experiments. 


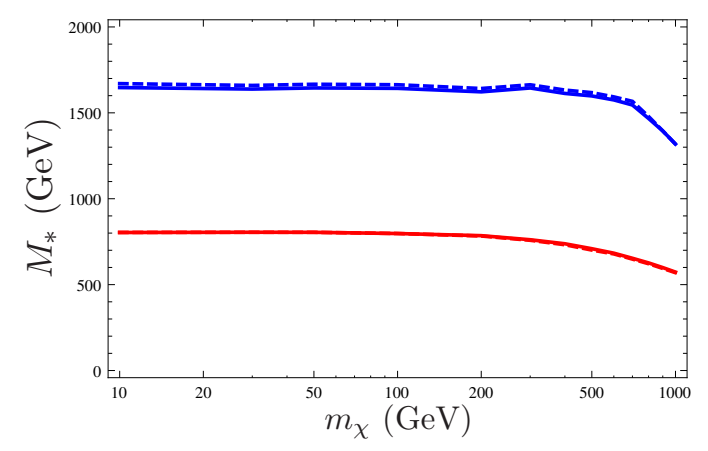

(a)

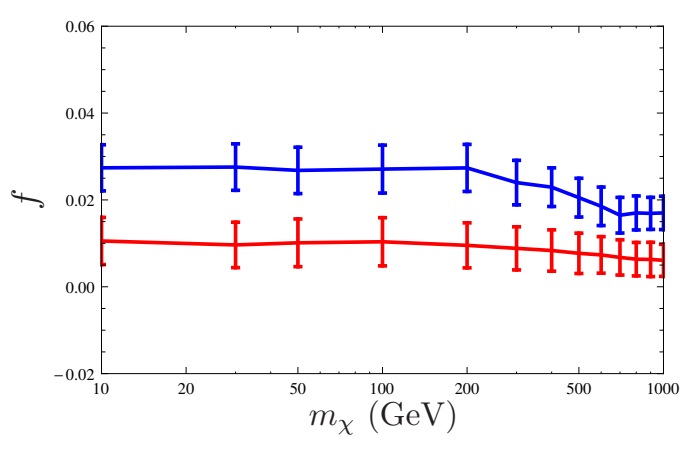

(b)

Figure 7. (a) Shown is the discovery reach for the operators QDM (blue) and GDM (red) in the presence of background at the $14 \mathrm{TeV}$ LHC at $100 \mathrm{fb}^{-1}$, with only statistical errors using a counting experiment alone (solid lines) and with JEPs (dashed lines). (b) For $M_{*}$ values at the discovery reach shown in (a) using the counting experiment alone, we show the separation in JEPs parameterized as $f$. We see that the QDM and GDM hypotheses are well separated by using the JEP.

Direct detection experiments look for recoiling nuclei from dark matter particles scattering off of a target. In the EFT formalism, the same operators which give dark matter production at the LHC (i.e. couplings to quarks and gluons) also give rise to scattering in direct detection experiments. Here, we shall only discuss spin-independent direct detection signals since the specific operators we have considered for QDM and GDM only give rise to these kind of interactions.

Given an EFT of dark matter scattering with quarks or gluons, there are two parameters characterizing the EFT, $m_{\chi}$ and $M_{*}$, and signals in direct detection experiments and the LHC can be related for each values of these parameters. However, as can be seen from figure 8, different EFT operators can give widely different predictions for direct detection. Therefore, by the counting experiment alone, it is not possible to directly relate signals at the LHC and direct detection.

We have shown in this work that JEPs can be used to discriminate between the QDM and GDM EFTs at the LHC. This identification of whether the monojet signal is arising from a QDM or GDM EFT can have two possible implications for the connection to direct detection experiments depending upon the relevant parameter space region:

1. The dark matter model is allowed by direct detection constraints. We can see that for the QDM signal this is only true for very low dark matter masses, but for the GDM signal, a wide range of masses can be consistent with direct detection bounds. Knowledge of the specific EFT responsible for the monojet signal will then predict the rates and spectra expected at direct detection experiments and help us to design future direct detection experiments to probe the relevant parameter space.

2. The particular model is already ruled out by direct detection, and hence the particle discovered in monojets at the LHC does not constitute the local dark matter. This then points to long-lived particles, multiple components of dark matter, or some other suppression of scattering in direct detection experiments (e.g. inelastic dark matter). 


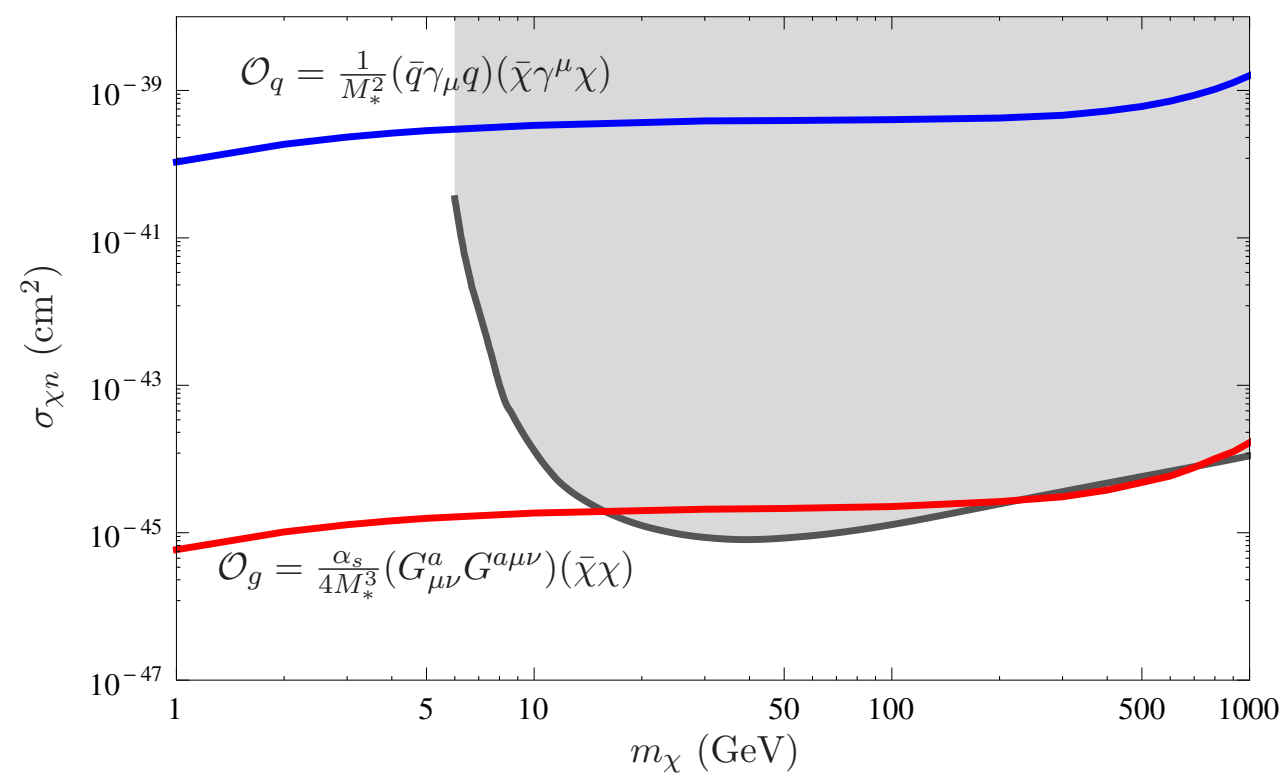

Figure 8. WIMP-nucleon scattering cross section from the QDM (blue) and GDM (red) models described in the text. For each dark matter mass, the value of $M_{*}$ is chosen at the $5 \sigma$ discovery reach limit at the $14 \mathrm{TeV}$ LHC, by using a counting experiment with $10 \mathrm{fb}^{-1}$ of luminosity. The gray shaded region shows the recent exclusion limits from LUX [13].

Thus, the identification of the specific EFT using JEPs is a valuable and necessary tool when correlating the results of monojet searches with direct detection experiments. We note that not all EFTs can necessarily be differentiated in this way, and for a complete picture it might be necessary to use information from complementary searches.

\section{Discussion and conclusions}

In this paper, we studied monojet searches for dark matter at the LHC. For simplicity we focused on effective field theories for dark matter interactions with quarks (QDM) and gluons (GDM). We found that the monojet associated with each of these EFTs has a characteristic quark/gluon fraction initiating the jet at the parton level. Moreover, the dominant backgrounds $(W, Z+$ jets), also have a characteristic gluon fraction, which is distinct from the signal.

We use the averaged JEP of the monojet event sample to measure the fraction of gluon-initiated jets. While the individual event JEPs can have large fluctuations on an event-by-event basis, the sample average JEP is very robust, and is less sensitive to the shape of the underlying fluctuations.

The use of the averaged JEP along with the event counts provides us with better sensitivity than just using a counting experiment alone to discriminate between signal and background. We found that when the counting experiment sensitivity has large systematic errors, the additional use of JEP discrimination yields a significant improvement in our ability to set limits or discover an excess in monojets at the LHC.

In the event of a discovery of an excess in monojets, JEPs become an invaluable tool to identify the specific dark matter interactions (separating QDM from GDM). 
The EFT approach to dark matter interactions connects signals at the LHC with direct detection experiments. However, it does so only after the specific effective operator for interaction between the dark matter and quarks or gluons is identified. Different operators which give the same number of events at the LHC can give predictions spanning five orders of magnitude at direct detection experiments. Thus, identification of the specific QDM or GDM EFT is especially important when comparing the signals at the LHC with results from direct detection experiments.

Identification of the operator responsible for a monojet excess would sharply predict the expected signal region for direct detection experiments. A null result at direct detection experiments in the relevant region would point to the possibility that the particle discovered at the LHC is not one which makes up the local dark matter density.

In this work we only considered the specific operators $\mathcal{O}_{q}$ and $\mathcal{O}_{g}$ which give rise to very different gluon-fractions for the monojet and are thus best suited for discrimination using the technique of JEPs. One could consider other quark and gluon bilinears as well. As mentioned in section 2, the scalar quark bilinears give rise to very weak constraints and can be ignored for the purposes of monojet searches. However, two other cases of interest are axial-vector quark bilinears and non-flavor universal couplings to quarks. The axial-vector scenario gives practically identical cross-sections as the vector quark bilinear at the LHC, but when comparing to direct detection experiments, the relevant bounds arise from the spin-dependent interactions and this would alter the discussion presented in section 7. The JEP discrimination method proposed in this paper is mostly insensitive to non-universal flavor couplings to quarks, however, complementary searches such as monophotons/mono- $W /$ mono- $Z$ can probe such non-universal couplings.

Finally, we note that there are other methods that could be used to discriminate between the various DM EFTs. Event-by-event tagging of quark-initiated and gluon-initiated jets has been proposed (see $[69,70]$ and references therein), and this can also be used to separate operators with different gluon fractions for the monojet. There are also techniques beyond quark/gluon identification by which dark matter models can be distinguished in monojet searches. Kinematic distributions of the associated jets can potentially yield information about the specific dark matter operators involved. In [71], it was shown that azimuthal angle correlations between the two leading jets in $2 j+\bar{\chi} \chi$ events can be used to separate tree-level production from loop production of dark matter.

For future extensions of our work it would be interesting to combine our technique with these other discrimination techniques to reach the maximum sensitivity of monojet searches.

\section{Acknowledgments}

We would like to acknowledge useful discussions with Patrick Fox, Claudia Frugiuele, Roni Harnik, Sonia El Hedri, Raoul Röntsch, Ciaran Williams, and C.-P. Yuan. VR is supported by NSF Grant No. PHY-0855561. PA would like to acknowledge support by the National Science Foundation under Grant No. PHYS-1066293 and the hospitality of the Aspen Center for Physics, where a part of this work was completed. VR would like to thank 
Fermilab for hospitality during the completion of this work. Fermilab is operated by Fermi Research Alliance, LLC under Contract No. DE-AC02-07CH11359 with the United States Department of Energy.

Open Access. This article is distributed under the terms of the Creative Commons Attribution License (CC-BY 4.0), which permits any use, distribution and reproduction in any medium, provided the original author(s) and source are credited.

\section{References}

[1] G. Bertone, D. Hooper and J. Silk, Particle dark matter: Evidence, candidates and constraints, Phys. Rept. 405 (2005) 279 [hep-ph/0404175] [INSPIRE].

[2] P. Benetti et al., First results from a Dark Matter search with liquid Argon at $87 \mathrm{~K}$ in the Gran Sasso Underground Laboratory, Astropart. Phys. 28 (2008) 495 [astro-ph/0701286] [INSPIRE].

[3] CDMS-II collaboration, Z. Ahmed et al., Dark Matter Search Results from the CDMS II Experiment, Science 327 (2010) 1619 [arXiv:0912.3592] [INSPIRE].

[4] DAMA, LIBRA collaborations, R. Bernabei et al., New results from DAMA/LIBRA, Eur. Phys. J. C 67 (2010) 39 [arXiv: 1002.1028] [InSPIRE].

[5] D.Y. Akimov et al., WIMP-nucleon cross-section results from the second science run of ZEPLIN-III, Phys. Lett. B 709 (2012) 14 [arXiv:1110.4769] [InSPIRE].

[6] G. Angloher et al., Results from $730 \mathrm{~kg}$ days of the CRESST-II Dark Matter Search, Eur. Phys. J. C 72 (2012) 1971 [arXiv:1109.0702] [INSPIRE].

[7] EDELWEISS collaboration, E. Armengaud et al., A search for low-mass WIMPs with EDELWEISS-II heat-and-ionization detectors, Phys. Rev. D 86 (2012) 051701 [arXiv:1207.1815] [INSPIRE].

[8] CoGeNT collaboration, C.E. Aalseth et al., CoGeNT: A Search for Low-Mass Dark Matter using p-type Point Contact Germanium Detectors, Phys. Rev. D 88 (2013) 012002 [arXiv: 1208.5737] [INSPIRE].

[9] S.C. Kim et al., New Limits on Interactions between Weakly Interacting Massive Particles and Nucleons Obtained with CsI(Tl) Crystal Detectors, Phys. Rev. Lett. 108 (2012) 181301 [arXiv:1204.2646] [INSPIRE].

[10] COUPP collaboration, E. Behnke et al., First Dark Matter Search Results from a 4-kg $\mathrm{CF}_{3} \mathrm{I}$ Bubble Chamber Operated in a Deep Underground Site, Phys. Rev. D 86 (2012) 052001 [arXiv: 1204.3094] [INSPIRE].

[11] XENON collaboration, L. Baudis, Results from the XENON100 Dark Matter Search Experiment, arXiv: 1203.1589 [INSPIRE].

[12] CDMS collaboration, R. Agnese et al., Silicon Detector Dark Matter Results from the Final Exposure of CDMS II, Phys. Rev. Lett. 111 (2013) 251301 [arXiv:1304.4279] [INSPIRE].

[13] LUX collaboration, D.S. Akerib et al., First results from the LUX dark matter experiment at the Sanford Underground Research Facility, Phys. Rev. Lett. 112 (2014) 091303 [arXiv: 1310.8214] [INSPIRE]. 
[14] HEAT collaboration, S.W. Barwick et al., Measurements of the cosmic ray positron fraction from 1-GeV to 50-GeV, Astrophys. J. 482 (1997) L191 [astro-ph/9703192] [INSPIRE].

[15] Super-Kamiokande collaboration, S. Desai et al., Search for dark matter WIMPs using upward through-going muons in Super-Kamiokande, Phys. Rev. D 70 (2004) 083523 [Erratum ibid. D 70 (2004) 109901] [hep-ex/0404025] [INSPIRE].

[16] AMS-01 collaboration, M. Aguilar et al., Cosmic-ray positron fraction measurement from 1 to 30-GeV with AMS-01, Phys. Lett. B 646 (2007) 145 [astro-ph/0703154] [INSPIRE].

[17] J. Chang et al., An excess of cosmic ray electrons at energies of 300-800 GeV, Nature 456 (2008) 362 [INSPIRE].

[18] Fermi LAT collaboration, M. Ackermann et al., Measurement of separate cosmic-ray electron and positron spectra with the Fermi Large Area Telescope, Phys. Rev. Lett. 108 (2012) 011103 [arXiv:1109.0521] [INSPIRE].

[19] ICECuBe collaboration, M.G. Aartsen et al., Search for dark matter annihilations in the Sun with the 79-string IceCube detector, Phys. Rev. Lett. 110 (2013) 131302 [arXiv:1212.4097] [INSPIRE].

[20] Fermi LAT collaboration, G. Zaharijas, J. Conrad, A. Cuoco and Z. Yang, Constraints on the Galactic Dark Matter signal from the Fermi-LAT measurement of the diffuse gamma-ray emission, eConf C 121028 (2012) 183 [arXiv:1304.2547] [INSPIRE].

[21] PAMELA collaboration, O. Adriani et al., Cosmic-Ray Positron Energy Spectrum Measured by PAMELA, Phys. Rev. Lett. 111 (2013) 081102 [arXiv:1308.0133] [inSPIRE].

[22] Fermi-LAT collaboration, M. Ackermann et al., Search for Gamma-ray Spectral Lines with the Fermi Large Area Telescope and Dark Matter Implications, Phys. Rev. D 88 (2013) 082002 [arXiv: 1305. 5597] [INSPIRE].

[23] Fermi-LAT collaboration, M. Ackermann et al., Dark Matter Constraints from Observations of 25 Milky Way Satellite Galaxies with the Fermi Large Area Telescope, Phys. Rev. D 89 (2014) 042001 [arXiv: 1310.0828] [INSPIRE].

[24] OPAL collaboration, G. Abbiendi et al., Photonic events with missing energy in $e^{+} e^{-}$ collisions at $\sqrt{s}=189 \mathrm{GeV}$, Eur. Phys. J. C 18 (2000) 253 [hep-ex/0005002] [INSPIRE].

[25] ALEPH collaboration, A. Heister et al., Single photon and multiphoton production in $e^{+} e^{-}$ collisions at $\sqrt{s}$ up to $209 \mathrm{GeV}$, Eur. Phys. J. C 28 (2003) 1 [INSPIRE].

[26] D0 collaboration, V.M. Abazov et al., Search for large extra dimensions in the monojet + missing $E_{T}$ channel at DØ, Phys. Rev. Lett. 90 (2003) 251802 [hep-ex/0302014] [INSPIRE].

[27] DELPHI collaboration, J. Abdallah et al., Photon events with missing energy in $e^{+} e^{-}$ collisions at $\sqrt{s}=130 \mathrm{GeV}$ to $209 \mathrm{GeV}$, Eur. Phys. J. C 38 (2005) 395 [hep-ex/0406019] [INSPIRE].

[28] L3 collaboration, P. Achard et al., Single photon and multiphoton events with missing energy in $e^{+} e^{-}$collisions at LEP, Phys. Lett. B 587 (2004) 16 [hep-ex/0402002] [INSPIRE].

[29] DELPHI collaboration, J. Abdallah et al., Search for one large extra dimension with the DELPHI detector at LEP, Eur. Phys. J. C 60 (2009) 17 [arXiv:0901.4486] [INSPIRE].

[30] ATLAS collaboration, Search for new phenomena with the monojet and missing transverse momentum signature using the ATLAS detector in $\sqrt{s}=7 \mathrm{TeV}$ proton-proton collisions, Phys. Lett. B 705 (2011) 294 [arXiv:1106.5327] [INSPIRE]. 
[31] CDF collaboration, T. Aaltonen et al., Search for a dark matter candidate produced in association with a single top quark in p $\bar{p}$ collisions at $\sqrt{s}=1.96 \mathrm{TeV}$, Phys. Rev. Lett. 108 (2012) 201802 [arXiv:1202.5653] [INSPIRE].

[32] CDF collaboration, T. Aaltonen et al., A search for dark matter in events with one jet and missing transverse energy in $p \bar{p}$ collisions at $\sqrt{s}=1.96$ TeV, Phys. Rev. Lett. 108 (2012) 211804 [arXiv: 1203.0742] [INSPIRE].

[33] ATLAS collaboration, Search for dark matter candidates and large extra dimensions in events with a jet and missing transverse momentum with the ATLAS detector, JHEP 04 (2013) 075 [arXiv: 1210.4491] [INSPIRE].

[34] CMS collaboration, Search for dark matter and large extra dimensions in monojet events in pp collisions at $\sqrt{s}=7 \mathrm{TeV}$, JHEP 09 (2012) 094 [arXiv:1206.5663] [INSPIRE].

[35] CMS collaboration, Search for Dark Matter and Large Extra Dimensions in pp Collisions Yielding a Photon and Missing Transverse Energy, Phys. Rev. Lett. 108 (2012) 261803 [arXiv:1204.0821] [INSPIRE].

[36] ATLAS collaboration, Search for dark matter in events with a hadronically decaying $W$ or $Z$ boson and missing transverse momentum in pp collisions at $\sqrt{s}=8$ TeV with the ATLAS detector, Phys. Rev. Lett. 112 (2014) 041802 [arXiv: 1309.4017] [INSPIRE].

[37] C. Han et al., Probing Light Higgsinos in Natural SUSY from Monojet Signals at the LHC, JHEP 02 (2014) 049 [arXiv: 1310.4274] [INSPIRE].

[38] A. Alves, S. Profumo and F.S. Queiroz, The dark $Z^{\prime}$ portal: direct, indirect and collider searches, JHEP 04 (2014) 063 [arXiv: 1312.5281] [INSPIRE].

[39] J. Goodman et al., Constraints on Light Majorana dark Matter from Colliders, Phys. Lett. B 695 (2011) 185 [arXiv: 1005.1286] [INSPIRE].

[40] J. Goodman et al., Constraints on Dark Matter from Colliders, Phys. Rev. D 82 (2010) 116010 [arXiv: 1008.1783] [INSPIRE].

[41] Y. Bai, P.J. Fox and R. Harnik, The Tevatron at the Frontier of Dark Matter Direct Detection, JHEP 12 (2010) 048 [arXiv:1005.3797] [INSPIRE].

[42] A. Rajaraman, W. Shepherd, T.M.P. Tait and A.M. Wijangco, LHC Bounds on Interactions of Dark Matter, Phys. Rev. D 84 (2011) 095013 [arXiv:1108.1196] [INSPIRE].

[43] P.J. Fox, R. Harnik, J. Kopp and Y. Tsai, Missing Energy Signatures of Dark Matter at the LHC, Phys. Rev. D 85 (2012) 056011 [arXiv:1109.4398] [InSPIRE].

[44] I.M. Shoemaker and L. Vecchi, Unitarity and Monojet Bounds on Models for DAMA, CoGeNT and CRESST-II, Phys. Rev. D 86 (2012) 015023 [arXiv:1112.5457] [InSPIRE].

[45] S. Chang, R. Edezhath, J. Hutchinson and M. Luty, Effective WIMPs, Phys. Rev. D 89 (2014) 015011 [arXiv:1307.8120] [INSPIRE].

[46] Y. Bai and J. Berger, Fermion Portal Dark Matter, JHEP 11 (2013) 171 [arXiv:1308.0612] [INSPIRE].

[47] A. DiFranzo, K.I. Nagao, A. Rajaraman and T.M.P. Tait, Simplified Models for Dark Matter Interacting with Quarks, JHEP 11 (2013) 014 [arXiv:1308.2679] [INSPIRE].

[48] O. Buchmueller, M.J. Dolan and C. McCabe, Beyond Effective Field Theory for Dark Matter Searches at the LHC, JHEP 01 (2014) 025 [arXiv:1308.6799] [INSPIRE]. 
[49] N. Zhou, D. Berge and D. Whiteson, Mono-everything: combined limits on dark matter production at colliders from multiple final states, Phys. Rev. D 87 (2013) 095013 [arXiv: 1302.3619] [INSPIRE].

[50] Y. Gershtein, F. Petriello, S. Quackenbush and K.M. Zurek, Discovering hidden sectors with mono-photon Z'o searches, Phys. Rev. D 78 (2008) 095002 [arXiv:0809.2849] [INSPIRE].

[51] Y. Bai and T.M.P. Tait, Searches with Mono-Leptons, Phys. Lett. B 723 (2013) 384 [arXiv: 1208.4361] [INSPIRE].

[52] N.F. Bell et al., Searching for Dark Matter at the LHC with a Mono-Z, Phys. Rev. D 86 (2012) 096011 [arXiv: 1209.0231] [INSPIRE].

[53] L.M. Carpenter, A. Nelson, C. Shimmin, T.M.P. Tait and D. Whiteson, Collider searches for dark matter in events with a $Z$ boson and missing energy, Phys. Rev. D 87 (2013) 074005 [arXiv: 1212.3352] [INSPIRE].

[54] A.A. Petrov and W. Shepherd, Searching for dark matter at LHC with Mono-Higgs production, Phys. Lett. B 730 (2014) 178 [arXiv:1311.1511] [INSPIRE].

[55] L. Carpenter et al., Mono-Higgs: a new collider probe of dark matter, Phys. Rev. D 89 (2014) 075017 [arXiv: 1312.2592] [INSPIRE].

[56] ATLAS collaboration, Search for New Phenomena in Monojet plus Missing Transverse Momentum Final States using $10 \mathrm{fb}-1$ of pp Collisions at $\sqrt{s}=8$ TeV with the ATLAS detector at the LHC, ATLAS-CONF-2012-147 (2012).

[57] CMS collaboration, Search for new physics in monojet events in pp collisions at $\sqrt{s}=8 \mathrm{TeV}$, CMS-PAS-EXO-12-048.

[58] M. Cacciari, G.P. Salam and G. Soyez, The anti-k(t) jet clustering algorithm, JHEP 04 (2008) 063 [arXiv: 0802.1189] [INSPIRE].

[59] T. Sjöstrand, S. Mrenna and P.Z. Skands, A Brief Introduction to PYTHIA 8.1, Comput. Phys. Commun. 178 (2008) 852 [arXiv:0710.3820] [INSPIRE].

[60] CDF collaboration, D. Acosta et al., Study of jet shapes in inclusive jet production in $p \bar{p}$ collisions at $\sqrt{s}=1.96 \mathrm{TeV}$, Phys. Rev. D 71 (2005) 112002 [hep-ex/0505013] [InSPIRE].

[61] ATLAS collaboration, Study of Jet Shapes in Inclusive Jet Production in pp Collisions at $\sqrt{s}=7 \mathrm{TeV}$ using the ATLAS Detector, Phys. Rev. D 83 (2011) 052003 [arXiv:1101.0070] [INSPIRE].

[62] CMS collaboration, Hadronic Event Shapes in pp Collisions at 7 TeV, CMS-PAS-QCD-10-013 (2010).

[63] H.-n. Li, Z. Li and C.-P. Yuan, QCD resummation for jet substructures, Phys. Rev. Lett. 107 (2011) 152001 [arXiv:1107.4535] [INSPIRE].

[64] H.-n. Li, Z. Li and C.-P. Yuan, QCD resummation for light-particle jets, Phys. Rev. D 87 (2013) 074025 [arXiv: 1206.1344] [INSPIRE].

[65] J. Alwall, M. Herquet, F. Maltoni, O. Mattelaer and T. Stelzer, MadGraph 5: Going Beyond, JHEP 06 (2011) 128 [arXiv:1106.0522] [INSPIRE].

[66] P.-A. Delsart, K.L. Geerlings, J. Huston, B.T. Martin and C.K. Vermilion, SpartyJet 4.0 User's Manual, arXiv:1201.3617 [INSPIRE].

[67] M. Cacciari, G.P. Salam and G. Soyez, FastJet User Manual, Eur. Phys. J. C 72 (2012) 1896 [arXiv: 1111.6097] [INSPIRE]. 
[68] U. Haisch, F. Kahlhoefer and E. Re, QCD effects in mono-jet searches for dark matter, JHEP 12 (2013) 007 [arXiv: 1310.4491] [INSPIRE].

[69] J. Gallicchio and M.D. Schwartz, Quark and Gluon Jet Substructure, JHEP 04 (2013) 090 [arXiv: 1211.7038] [INSPIRE].

[70] J. Gallicchio and M.D. Schwartz, Quark and Gluon Tagging at the LHC, Phys. Rev. Lett. 107 (2011) 172001 [arXiv: 1106.3076] [INSPIRE].

[71] U. Haisch, A. Hibbs and E. Re, Determining the structure of dark-matter couplings at the LHC, Phys. Rev. D 89 (2014) 034009 [arXiv:1311.7131] [InSPIRE]. 